\title{
Competitive Exclusion Prevents Colonization and Compartmentalization Reduces Transmission of ESBL-Producing Escherichia coli in Broilers
}

OPEN ACCESS

Edited by:

David Rodriguez-Lazaro,

University of Burgos, Spain

Reviewed by:

Gilles Salvat,

Agence Nationale de Sécurité

Sanitaire de l'Alimentation,

de l'Environnement et du Travail

(ANSES), France

Héctor Argüello,

University of Córdoba, Spain

*Correspondence:

Anita Dame-Korevaar

m.a.damekorevaar@gmail.com

${ }^{\dagger}$ Present address:

Daniela Ceccarelli,

Research Executive Agency, European Commission, Brussels,

Belgium

Specialty section:

This article was submitted to

Food Microbiology,

a section of the journal

Frontiers in Microbiology

Received: 28 May 2020

Accepted: 21 October 2020

Published: 10 November 2020

Citation:

Dame-Korevaar A, Kers JG,

van der Goot J, Velkers FC, Ceccarelli D, Mevius DJ, Stegeman A and Fischer EAJ (2020) Competitive

Exclusion Prevents Colonization and Compartmentalization Reduces Transmission of ESBL-Producing

Escherichia coli in Broilers.

Front. Microbiol. 11:566619. doi: 10.3389/fmicb.2020.566619
Anita Dame-Korevaar ${ }^{1,2 *}$, Jannigje G. Kers ${ }^{1,3}$, Jeanet van der Goot ${ }^{2}$, Francisca C. Velkers ${ }^{1}$, Daniela Ceccarelli2t, Dik J. Mevius ${ }^{2,4}$, Arjan Stegeman ${ }^{1}$ and Egil A. J. Fischer ${ }^{1}$

'Department of Farm Animal Health, Faculty of Veterinary Medicine, Utrecht University, Utrecht, Netherlands, ${ }^{2}$ Department of Bacteriology and Epidemiology, Wageningen Bioveterinary Research, Lelystad, Netherlands, ${ }^{3}$ Laboratory of Microbiology, Wageningen University, Wageningen, Netherlands, ${ }^{4}$ Department of Infectious Diseases and Immunology, Faculty of Veterinary Medicine, Utrecht University, Utrecht, Netherlands

Extended spectrum beta-lactamase (ESBL)-producing bacteria are resistant to extended-spectrum cephalosporins and are common in broilers. Interventions are needed to reduce the prevalence of ESBL-producing bacteria in the broiler production pyramid. This study investigated two different interventions. The effect of a prolonged supply of competitive exclusion (CE) product and compartmentalization on colonization and transmission, after challenge with a low dose of ESBL-producing Escherichia coli, in broilers kept under semi-field conditions, were examined. One-day-old broilers (Ross 308) ( $n=400$ ) were housed in four experimental rooms, subdivided in one seeder (S/C1)-pen and eight contact (C2)-pens. In two rooms, CE product was supplied from day 0 to 7 . At day 5 , seeder-broilers were inoculated with $E$. coli strain carrying bla $_{C T X-M-1}$ on plasmid Incl1 (CTX-M-1-E. coli). Presence of CTX-M-1-E. coli was determined using cloacal swabs (day 5-21 daily) and cecal samples (day 21). Time until colonization and cecal excretion $\left(\log _{10} \mathrm{CFU} / \mathrm{g}\right)$ were analyzed using survival analysis and linear regression. Transmission coefficients within and between pens were estimated using maximum likelihood. The microbiota composition was assessed by $16 S$ ribosomal RNA gene amplicon sequencing in cecal content of broilers on days 5 and 21. None of the CE broilers was CTX-M-1-E. coli positive. In contrast, in the untreated rooms 187/200 of the broilers were CTX-M-1-E. coli positive at day 21 . Broilers in C2-pens were colonized later than seeder-broilers (Time to event Ratio 3.53, 95\% Cl 3.14 to 3.93). The transmission coefficient between pens was lower than within pens $\left(3.28 \times 10^{-4}\right.$ day $^{-2}, 95 \% \mathrm{Cl} 2.41 \times 10^{-4}$ to $4.32 \times 10^{-4}$ vs. $6.12 \times 10^{-2}$ day $^{-2}, 95 \% \mathrm{Cl} 4.78 \times 10^{-2}$ to $\left.7.64 \times 10^{-2}\right)$. The alpha diversity of the cecal microbiota content was higher in CE broilers than in control broilers at days 5 and 21 . The supply of a CE product from day 0 to 7 prevented colonization of CTX-M-1-E. coli after challenge at day 5 , likely as a result of CE induced effects on the 
microbiota composition. Furthermore, compartmentalization reduced transmission rate between broilers. Therefore, a combination of compartmentalization and supply of a CE product may be a useful intervention to reduce transmission and prevent colonization of $\mathrm{ESBL} / \mathrm{pAmpC}$-producing bacteria in the broiler production pyramid.

Keywords: poultry, compartments, intervention, antimicrobial resistance, ESBL, Escherichia coli, colonization

\section{INTRODUCTION}

Extended spectrum beta-lactamase and plasmid AmpC betalactamase (ESBL/pAmpC)-producing bacteria are resistant to extended-spectrum cephalosporins (ESC). ESBL/pAmpCproducing bacteria are present in humans, animals and the environment (Blaak et al., 2015). Poultry is known as a source of $\mathrm{ESBL} / \mathrm{pAmpC}$-producing bacteria and high prevalence in poultry and poultry products have been reported in several European countries, as reviewed by Saliu et al. (2017). ESBL/pAmpCproducing bacteria are present at all levels of the broiler production pyramid (Dierikx et al., 2013; Agerso et al., 2014; Nilsson et al., 2014; Zurfluh et al., 2014a,b; Projahn et al., 2018). Different routes of transmission within the broiler production pyramid have been described, for example between generations, via the hatcheries, and on and between farms (Dame-Korevaar et al., 2019a). As a consequence, introduction of ESBL/pAmpCproducing bacteria can occur at several levels of the broiler production pyramid, for example at the farm or at the hatchery. A recent study estimated that, based on the proportional similarity index (PSI), the average transfer of ESBL/pAmpC genes between subsequent generations in the broiler production pyramid is almost 50\% (Apostolakos et al., 2019). However, for most of the routes it is unknown to what extent they contribute to the presence of ESBL/pAmpC-producing bacteria in the broiler production pyramid. In Netherlands, antimicrobial resistance in broilers has decreased significantly since 2010 (Hesp et al., 2019), following the trend of reduced antimicrobial usage. However, additional interventions are needed to further reduce this prevalence in the broiler production pyramid.

Interventions can aim to reduce exposure of broilers to ESBL/pAmpC-producing Escherichia coli. This can be done by improving biosecurity. For example hygiene barriers can help reduce exposure to bacteria from the farm environment, or by cleaning and disinfection between production rounds. However, even after cleaning and disinfection, ESBL/pAmpCproducing bacteria might remain in the poultry house and result in colonization of the new flock (Daehre et al., 2018). In addition, housing measures may reduce the prevalence of ESBL/pAmpC-producing $E$. coli in poultry flocks. In turkeys, subdividing the flock was associated with a reduced risk for the presence of resistant $E$. coli on the farm (Jones et al., 2013). Experimental studies showed that spatial separation between infectious and susceptible animals reduced the transmission rate of Campylobacter in broilers (van Bunnik et al., 2012) and Streptococcus suis in pigs (Dekker et al., 2013). Further, interventions aiming at preventing colonization by $\mathrm{ESBL} / \mathrm{pAmpC}$-producing $E$. coli in broilers have been described, such as acid-based feed additives (Roth et al., 2017) or competitive exclusion (CE) products (Nuotio et al., 2013; Ceccarelli et al., 2017; Methner et al., 2019; Dame-Korevaar et al., 2020). CE products are aimed at establishing a natural community of intestinal bacteria to protect broilers from colonization by invaders (Nurmi et al., 1992). In modern broiler production, due to strict hygiene practices in commercial hatcheries, the initial bacterial load to colonize the chicken intestinal tract shortly after hatch is low (Varmuzova et al., 2016; Donaldson et al., 2017). Eggs are usually disinfected to remove bacterial contamination before placement in the hatcher. Consequently, the chicks are exposed mostly to bacteria from environmental sources rather than parental sources upon hatching. Microbial treatment supplied after hatch has been shown to affect the development of bacterial taxa found in growing chickens (Ballou et al., 2016; Schokker et al., 2017). This suggests that early supply of CE products might influence microbiota composition and act as a possible intervention to prevent colonization by ESBL/pAmpC-producing $E$. coli in young broilers. A single supply of CE product before challenge with a high dose of ESBL-producing E. coli has already showed to reduce colonization, cecal and fecal excretion $(\mathrm{CFU} / \mathrm{g})$, as well as transmission of ESBL-producing E. coli (Nuotio et al., 2013; Ceccarelli et al., 2017; Methner et al., 2019). Additionally, $\mathrm{CE}$ products resulted in a reduced intestinal and cecal excretion (CFU/g) after challenge with pathogenic E. coli (Hofacre et al., 2002). A prolonged supply of CE product via the drinking water to broilers kept in isolators, from day of hatch until day 14 resulted in a delay and even prevention of colonization after challenge with a in the field realistic low dose of ESBL-producing E. coli (Dame-Korevaar et al., 2020).

The aim of this study was to determine the effect of interventions on colonization and transmission of ESBLproducing E. coli in young broiler chicks kept under semi-field circumstances. Two interventions were included: (1) prolonged supply of CE product from day of hatch until day 7, and (2) compartmentalization of a broiler flock. To investigate the effect of CE product on microbial composition, microbiota in cecal content was assessed before and after challenge by $16 \mathrm{~S}$ ribosomal RNA (rRNA) gene amplicon sequencing.

\section{MATERIALS AND METHODS}

\section{Ethics of Experimentation}

Broilers were observed daily and the presence of clinical signs, abnormal behavior and mortality was recorded. The study protocol was approved by the Dutch Central Authority for Scientific Procedures on Animals and the Animal Experiments 
Committee of Utrecht University (Utrecht, Netherlands) under registration number AVD108002015314; all procedures were done in full compliance with Dutch legislation, and is thus compliant with legislation in the EU directive 2016/3/EU.

\section{Birds, Housing and Management}

Conventional broiler chicks (Ross $308, n=416$ ), from a parent stock flock of 37 weeks of age, were transported directly after hatch to the animal facilities (Utrecht University, Utrecht, Netherlands). Upon arrival, the broilers were individually tagged, weighed, and randomly divided over four experimental rooms ( $n=104$ broilers per room). Each room was subdivided into nine pens, with one seeder (S/C1)-pen in the middle $\left(2 \mathrm{~m}^{2}, n=24\right.$ broilers), surrounded by eight contact (C2)-pens $\left(1 \mathrm{~m}^{2}, n=10\right.$ broilers per pen) (Figure 1). The $\mathrm{S} / \mathrm{C} 1$-pen was separated from the $\mathrm{C} 2$-pens by a mesh panel $(30 \mathrm{~cm}$ solid panel at the bottom, $40 \mathrm{~cm}$ mesh panel, $10 \mathrm{~cm}$ solid panel on top). Feed and water systems were also separated, and strict hygiene measures between pens were taken. No direct contact between the broilers was possible, but small particles (e.g., litter, dust) could be transferred between pens potentially. The $\mathrm{C} 2$-pens were separated from each other with wooden panels of $80 \mathrm{~cm}$ height, assuming no contact and no spread of particles was possible. At day 5, just before challenge with ESBL-producing $E$. coli, the number of broilers in the S/C1-pen was reduced to 20 , by removing the surplus broilers. Ten of the remaining 20 broilers in each S/C1-pen were randomly selected and transported to four separate isolators. In these isolators, the broilers (seeder (S) broilers) were inoculated with CTX-M-1-E. coli and after $1 \mathrm{~h}$ moved back to the original S/C1-pens (see section "E. coli Challenge"). Before the start of the experiment the parent flock, hatchery and research facilities were tested for the absence of ESBL/pAmpC-producing bacteria.

Broilers were housed on fine wood shavings. A standard broiler diet without any antibiotics or coccidiostats, radiated with 9 Gy, was available ad libitum. The intervention was supplied in the drinking water (see section "Intervention Competitive Exclusion"); therefore, drinking water was not

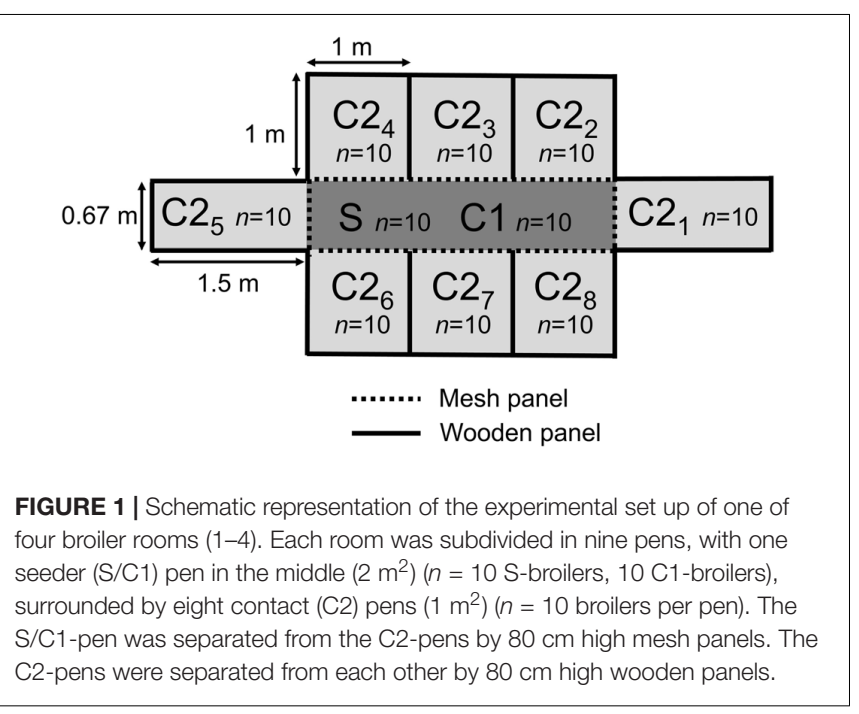

available ad libitum during the first seven days of the experiment in both intervention and control groups. Five out of 400 broilers died or were euthanized before the end of the experiment due to causes unrelated to the experiment, but by common causes in young broilers, i.e., not starting to eat or drink or omphalitis.

\section{Intervention Competitive Exclusion}

In two of four rooms a CE product was supplied, containing "natural, live intestinal microflora derived from specific pathogen free (SPF) chickens and manufactured by fermentation" (Aviguard $^{\circledR}$, MSD Animal Health, Netherlands). From the moment of arrival in the rooms (day 0, 10:00 a.m.) until day 7, (4:00 p.m.), CE product was supplied in the drinking water, twice per day. Water solutions containing the CE product were prepared in pre-dilution, with a dose level according to recommendations of the manufacturer, i.e., $0.125 \mathrm{~g}$ CE product per 10 broilers. The amount of drinking water was restricted between day 0 and 7 , based on the expected water consumption of 10 (C2-pen) and 20 (S/C1-pen) broilers in a pen to ensure that all supplied CE product would be consumed.

\section{E. coli Challenge}

Broilers were challenged with E. coli strain E38.27, which carries the ESBL gene bla $a_{C T X-M-1}$ on an IncI1 plasmid (CTX-M-1E. coli), isolated from conventional healthy broilers at slaughter age and resistant to cefotaxime (Dierikx et al., 2010). Oral inoculation of seeder (S) birds was performed on day 5 at 8:00 a.m. using a $1 \mathrm{~mL}$ syringe without a needle with $0.5 \mathrm{~mL}$ of $10^{2} \mathrm{CFU} / \mathrm{mL}$. The bacterial dilution was measured with the McFarland reader and retrospective colony counting. From $1 \mathrm{~h}$ after inoculation onward, 10 contact (C1) birds were exposed to 10 seeder birds, by moving the inoculated seeder birds to the corresponding S/C1-pens containing the contact birds.

\section{Cloacal and Cecal Samples}

Samples were taken using sterile dry cotton swabs (Copan 155C, Copan Diagnostics, United States). Broilers were sampled at day 5 at 4:00 a.m., just before inoculation to confirm absence of ESBL/pAmpC-producing bacteria, and from day 6 until day 21 daily at 8:00 a.m. At day 21, after the last sampling, post mortem examination was done within $30 \mathrm{~min}$ after euthanasia for each broiler. Broilers were weighed and sex was determined, exterior and interior abnormalities were assessed, and ceca were collected and stored on dry ice for further analysis.

\section{Microbiota Sample Collection and Analysis}

Cecal content samples were collected from five surplus broilers of the control group and from five surplus broilers of the CE intervention group $(n=10)$ at day 5 . At day 21 , cecal content of all broilers in the S/C1-pen in all four rooms $(n=80)$ was collected. The closed side of one of the two ceca was cut and cecal content was gently squeezed into a $2 \mathrm{~mL}$ sterile cryotube and snap frozen on dry ice and stored at $-80^{\circ} \mathrm{C}$ for genomic DNA extraction. To determine the microbial composition of the CE product, Aviguard ${ }^{\circledR}$ was suspended in PBS according to 
the manufacturer's instructions and four aliquots of $2 \mathrm{~mL}$ were stored at $-80^{\circ} \mathrm{C}$ for bacterial genomic DNA extraction. The full protocol for DNA extraction and determining microbiota composition was previously described (Kers et al., 2019). Briefly, DNA was extracted from $0.25 \mathrm{~g}$ cecal content or frozen CE product, using $700 \mu \mathrm{L}$ of Stool Transport and Recovery (STAR) buffer (Roche Diagnostics Nederland BV, Netherlands). All 94 samples were transferred to a sterile screw-capped $2 \mathrm{~mL}$ tube (BIOplastics BV, Netherlands), used for bead beating. The DNA concentrations were measured with a NanoDrop ND-1000 spectrophotometer (NanoDrop ${ }^{\circledR}$ Technologies, United States), and the DNA samples were stored at $-20^{\circ} \mathrm{C}$ until further use. Barcoded amplicons covering the variable regions V5-V6 and primers $784 \mathrm{~F}$ and $1064 \mathrm{R}$ were used for $16 \mathrm{~S}$ rRNA genebased microbial composition profiling as previously described (Ramiro-Garcia et al., 2016).

To ensure high quality sequencing data, synthetic communities of known composition were used as positive controls (Ramiro-Garcia et al., 2016) and nuclease free water as negative controls. Sequencing of resulting libraries was performed on Illumina Hiseq 2500 (Eurofins Genomics Germany $\mathrm{GmbH}$ ). The $16 \mathrm{~S}$ rRNA data was analyzed using NG-tax 2.0 (Ramiro-Garcia et al., 2016). In short, to generate amplicon sequence variants (ASVs), NG-Tax 2.0 employed a fast de novo ASV-picking algorithm. To assign taxonomy the SILVA 128 16S rRNA gene reference database was used (Quast et al., 2013). Bacterial names were identified on the genus-level, however, if this level was unknown, we used the lowest known taxonomic classification. Raw sequence data were deposited into the Sequence Read Archive (SRA) at the NCBI, under accession number PRJNA647260.

\section{ESBL-Producing E. coli Detection}

All cloacal samples were enriched in $3 \mathrm{~mL}$ Luria-Bertani (LB) broth. After overnight incubation at $37^{\circ} \mathrm{C}, 10 \mu \mathrm{L}$ broth were inoculated on MacConkey plates supplemented with $1 \mathrm{mg} / \mathrm{L}$ cefotaxime and incubated overnight at $37^{\circ} \mathrm{C}$. E. coli colonies growing on MacConkey plates supplemented with cefotaxime were referred to as CTX-M-1-E. coli. If visual assessment was not conclusive on the presence of E. coli, colonies were selected for further analyses using MALDI-TOF MS (Bruker Daltonik, Germany).

\section{ESBL-Producing E. coli and Total E. coli Quantification}

At day 21, content from one of two ceca of 80 selected broilers from rooms 1 and 2 was collected. For both rooms, selection included all broilers $(n=20)$ from the S/C1-pen and additionally 20 broilers from the C2-pens which were excreting CTX-M-1E. coli. Samples were processed as previously described (DameKorevaar et al., 2019b). Concentrations of ESBL-producing E. coli and total E. coli were determined semi-quantitatively. CFU/gram feces was calculated based on the highest dilution showing growth of typical E. coli colonies (Jett et al., 1997) and the weight of the feces on the swabs or the amount of cecal content collected (Ceccarelli et al., 2017). E. coli colonies growing on MacConkey plates supplemented with cefotaxime were referred to as CTXM-1-E. coli. If visual assessment was not conclusive on the presence of $E$. coli, colonies were selected for further analyses using MALDI-TOF MS.

\section{Statistical Analysis}

Statistical analyses were performed in R, version 3.4.3 (RStudio Team, 2016), using packages survival, phyloseq, microbiome, and vegan. Regressions were performed with function $\operatorname{glm}()$.

\section{Time Until Colonization}

Time until colonization was analyzed using parametric survival regression with an accelerated failure time model using a Weibull distribution (Kalbfleisch and Prentice, 2002). The hazard ratio was expected to be non-proportional during the experiment, because of the compartmentalization. This accelerated failure time model models the effect of the variables on the acceleration or deceleration of the time until colonization with CTX-M1-E. coli. Colonization of individual broilers was measured as excretion of CTX-M-1-E. coli and time until colonization was defined as the time point of the first cloacal swab of two consecutive cloacal swabs tested positive for CTX-M-1-E. coli. If the last swab (day 21) and the ceca tested positive, broilers were assumed to be colonized at day 21 . If only the ceca tested positive, broilers were not included as colonized birds within the time span of the experiment.

\section{Microbiota Composition}

Differences of relative abundance were tested with Wilcoxon rank-sum test and corrected for multiple testing using Benjamini-Hochberg $(\mathrm{BH})$ procedure. Alpha and beta diversity metrics were calculated and univariate and multivariate statistical analyses were applied to determine differences in the cecal microbiota. Alpha diversity (within sample richness) was determined using Faiths phylogenetic diversity, taking into account the phylogenetic relatedness (Faith, 2007). Differences in alpha diversity were tested using a nonparametric Kruskal-Wallis test. Beta diversity (between sample differences) was determined using weighted and unweighted UniFrac metrics (Lozupone et al., 2007). Principal coordinates analysis (PCoA) was used to visualize the data. To test differences within multivariate community data, non-parametric permutational analysis of variance (PERMANOVA) were used (Anderson, 2001).

\section{Transmission Coefficient}

The transmission coefficients for within and between pen transmission ( $\beta_{\text {within }}$ and $\left.\beta_{\text {between }}\right)$ were estimated based on a stochastic multi-pen SI model (Klinkenberg et al., 2002; Velthuis et al., 2007) in which the number of new cases is determined by transmission from excreting (I) birds to susceptible (S) birds for a total population of $(\mathrm{N})$ birds, using maximum likelihood estimation.

The probability $\left(p_{k}\right)$ for a susceptible animal in pen $k$ to become colonized during time interval $\Delta t$ was calculated based on the force of infection 
(foi) within the pen and between pens (S/C1-pen to C2-pen):

$$
p_{k}=1-e^{-\left(f o i_{\text {within }}+f o i_{\text {between }}\right) \Delta t} \quad \text { Equation } 1
$$

Two models were used in which the foi was based in model 1 on direct transmission or in model 2 on indirect transmission with a build-up of infectivity in the environment. In model 1 foi was determined by the proportion of excreting birds in the same pen $\left(\frac{I_{k}}{N_{k}}\right)$ and the proportions of excreting birds in the adjacent pen connected through a mesh panel $\left(\frac{I_{\text {adj }}}{N_{\text {adj }}}\right)$ during a time interval $\Delta t:$

$$
p_{k}=1-e^{-\left(\beta_{\text {within }} \frac{I_{k}}{N_{k}}+\beta_{\text {between }} \frac{I_{\text {adj }}}{N_{\text {adj }}}\right) \Delta t} \quad \text { Equation2 }
$$

The unit of $\beta_{\text {within }}$ and $\beta_{\text {between }}$ in model 1 is $1 /$ day, and is interpreted as the number of new colonized broilers per day, due to one positive broiler.

In model 2 the foi in pen $k$ was assumed to be a result of a build-up of infectivity in the environment. The cumulative sum of hours that all excreting birds were excreting in a pen (cumexcrhours $\mathrm{k}_{\mathrm{k}}$ ) and in the adjacent pen connected with a mesh panel (cumexcrhours adj $_{\text {) }}$ up to the beginning of the interval was used as a measure for environmental accumulation:

\section{$p_{k}$}

$=1-e^{-\left(\beta_{\text {within }_{\text {cumexcrhours }}}+\beta_{\text {between }_{\text {cumexcrhours }}} \text { adj }_{j}\right) \Delta t}$

Equation3

The unit of $\beta_{\text {within }}$ and $\beta_{\text {between }}$ in model 2 is $1 /$ day $^{2}$ and is interpreted as the number of new colonized broilers per day, caused by each day that one positive broiler has been excreting CTX-M-1-E. coli (Dekker et al., 2013; Dame-Korevaar et al., 2020).

\section{Cecal Excretion Levels}

The differences in cecal content of total E. coli and CTXM-1-E. coli (CFU/g) were tested using a linear regression model including the variables room, pen, sex, weight at day of hatch, weight at day 21, type of bird (S, C1, C2) and time until colonization. The best fitting model was obtained by backward selection based on lowest AIC value. The correlation between cecal content of CTX-M-1E. coli and time until colonization was tested using Pearson's correlation coefficient.

\section{RESULTS}

\section{Time Until Colonization}

Broilers in the CE groups (room 3 and 4) were not colonized with CTX-M-1-E. coli. In the control groups all broilers in room $1(n=100)$, and $87 / 100$ broilers in room 2 were colonized at the end of the experiment (Figure 2). Time until colonization was delayed for broilers in room 2 compared to broilers in room 1 (Time Ratio (TR) 3.00, 95\% CI 1.82 to 4.95), and for C2 broilers compared to seeder broilers (TR 3.53, 95\% CI 3.14 to 3.93). No difference in time until colonization was observed between seeder and C1 broilers (TR 1.14, 95\% CI 1.00 to 1.30). Weight at day of hatch, weight at day 21 and sex did not influence time until colonization (Table 1).

\section{Microbiota Composition in Cecal Content}

The alpha diversity (phylogenetic diversity) was higher in cecal content samples of the broilers supplied with CE product (CE broilers) compared to the control broilers on day 5 and day 21 (Figure 3). On day 21 no differences in alpha diversity between the two intervention rooms were observed $\left(\mathrm{X}^{2}=1.90\right.$, $p=0.17)$, but the control broilers in room 1 had a lower alpha diversity than control broilers from room $2\left(\mathrm{X}^{2}=4.92\right.$, $p=0.03)$. Within rooms, no differences between seeder and
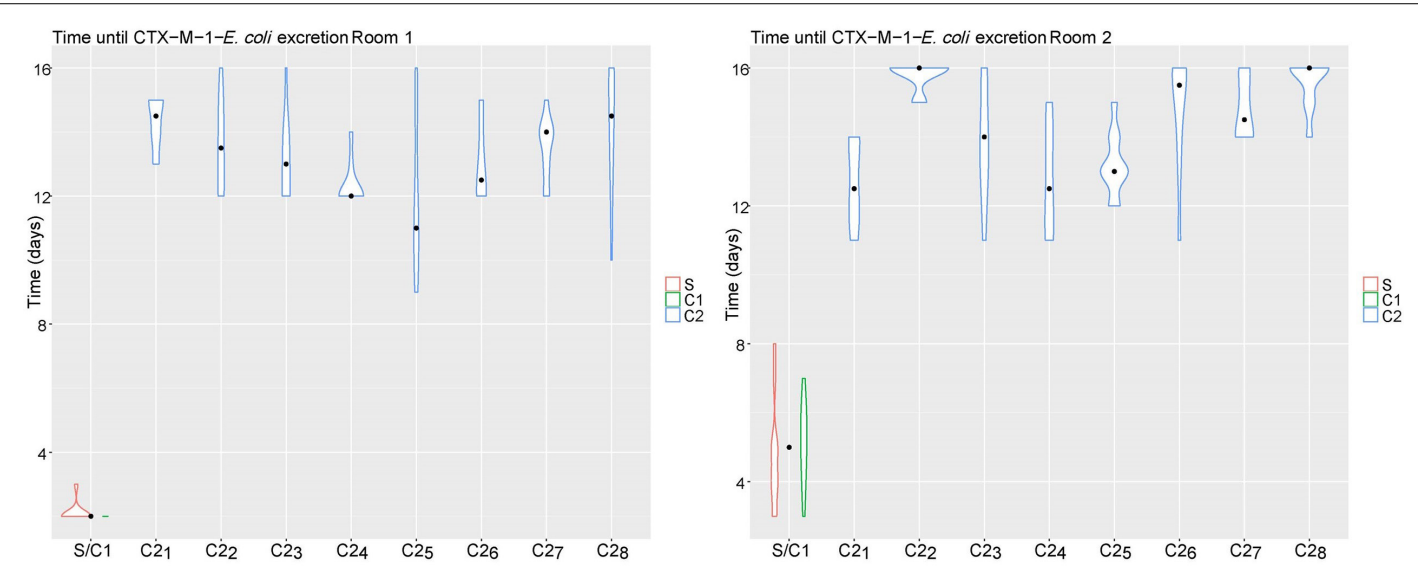

FIGURE 2 | Time until colonization (days) of CTX-M-1-E. coli per pen ( $\mathrm{S} / \mathrm{C} 1, \mathrm{C} 2{ }_{1}, \mathrm{C} 22_{2}, \mathrm{C} 2{ }_{3}, \mathrm{C} 2{ }_{4}, \mathrm{C} 22_{5}, \mathrm{C} 22_{6}, \mathrm{C} 27, \mathrm{C} 28$ ) and type of bird [seeder (S), contact 1 (C1), contact 2 (C2)] for room 1 (left) and room 2 (right). The violin plot indicates the total range of observations; the black dot indicates the median. 
TABLE 1 | Regression coefficients of time until colonization (95\% Cl) of CTX-M-1-E. coli for an accelerated failure time model.

\begin{tabular}{lcc}
\hline Variable & $\begin{array}{c}\text { Accelerated } \\
\text { failure time (days, } \\
\mathbf{9 5 \%} \mathbf{~ C l ) *}\end{array}$ \\
\hline Baseline survival (Room 1, Seeder, Male) & & $3.00(1.82-4.95)$ \\
Room 2 & Contact 1 & $1.24(1.15-1.33)$ \\
Animal type & Contact 2 & $3.53(3.14-3.93)$ \\
Sex & Female & $0.97(0.91-1.03)$ \\
Bodyweight at day 0 & & $1.00(0.99-1.02)$ \\
Bodyweight at day 21 & & $1.00(1.00-1.00)$ \\
\hline
\end{tabular}

${ }^{*}$ Accelerated failure time, indicating the acceleration or deceleration of the time until colonization with CTX-M-1-E. coli in the mentioned group compared to the reference (baseline) group. A ratio of $>1$ indicates a longer time until colonization, and a ratio of $<1$ indicates a shorter time until colonization.

contact (C1) broilers were found. Weight at day of hatch, weight at day 21 and sex did not influence the alpha diversity (data not shown).

In weighted and unweighted UniFrac (wuf and uf) distance based analysis, the supply of the CE product explained $60 \%$ (wuf) and 69\% (uf) of the variation between the cecal content samples on day 5 (Figure 4, principal coordinate analysis (PCoA), PERMANOVA, wuf: $R^{2}=0.598, p=0.009$, uf: $R^{2}=0.688$, $p=0.008)$. On day 21 , application of the CE product explained $46 \%$ (wuf) and 51\% (uf) of the variation between the cecal content samples (Figure 4, PERMANOVA, uf: $R^{2}=0.461$, $p=1.0 \times 10^{-4}$, wuf: $\left.R^{2}=0.510, p=1.0 \times 10^{-4}\right)$. Within rooms, being a seeder or contact broiler did not explain any of the variation between the cecal content samples. The variation between the two control groups was larger than between the two intervention rooms (wuf: $R^{2}=0.351$ vs. $\left.R^{2}=0.210\right)$.

The heatmap (Figure 5) shows all genera that significantly differed in relative abundance between CE broilers and control broilers at day 5 and 21. Selection of the first four clusters reveal two clusters with control broilers: one for the broilers of 5 days of age, and one for the broilers of 21 days of age. The other two clusters consist of CE broilers, one cluster contains broilers of both 5 and 21 days of age, while the second cluster contains only CE broilers of 21 days of age.

In the CE product, 22 different genera were identified (Table 2). Of these genera, five were more abundant in CE broilers than in control broilers at day 5: Collinsella, Eubacterium, Flavonifractor, Lachnoclostridium, and Lactobacillus. At day 21, genera Eubacterium coprostanoligenes, Bacteroides, Collinsella, Enterococcus, Eubacterium, Megamonas, Megasphaera, Slackia, and Sutterella were more abundant in CE than in control broilers (Table 2).

\section{Transmission}

Broilers in the CE groups (room 3 and 4) were not colonized with CTX-M-1-E. coli, and transmission was thus not observed.

In the control groups, the transmission coefficient between pens $\left(\beta_{\text {between }}\right)$ was lower than the transmission coefficient within pens $\left(\beta_{\text {within }}\right)$ for both models. Model 2 , with accumulated environmental transmission, was preferred over model 1, assuming direct transmission (AIC 402.1 vs. 438.1, Table 3). For model 2, $\beta_{\text {between }}$ was $3.28 \times 10^{-4}$ day $^{-2}\left(95 \%\right.$ CI $2.41 \times 10^{-4}$ to $\left.4.32 \times 10^{-4}\right)$ and $\beta_{\text {within }}$ was $6.12 \times 10^{-2}$ day $^{-2}\left(95 \%\right.$ CI $4.78 \times 10^{-2}$ to $7.64 \times 10^{-2}$ ) (Table 3).

\section{Cecal Excretion Levels}

Mean CTX-M-1-E. coli ( $\left.\log _{10} \mathrm{CFU} / \mathrm{g}\right)$ was lower in cecal samples from broilers from $\mathrm{C} 2$-pens than from the S/C1-pen, except for pen $\mathrm{C}_{2}$ and $\mathrm{C}_{7}$ (Table 4). CTX-M-1-E. coli $\left(\log _{10} \mathrm{CFU} / \mathrm{g}\right)$ was lower in cecal samples from broilers kept in room 2 than broilers kept in room 1 (estimate $-0.52,95 \%$ CI -0.91 to $-0.13 \log _{10}$ $\mathrm{CFU} / \mathrm{g}$ ). Broilers with a higher bodyweight at day of hatch had slightly higher cecal CTX-M-1-E. coli levels (estimate 0.08, 95\% CI 0.01 to $\left.0.15 \log _{10} \mathrm{CFU} / \mathrm{g}\right)$. Cecal CTX-M-1-E. coli levels were correlated with time until colonization, the shorter the time until colonization, the higher the cecal level $(r=-0.60,95 \%$ CI -0.73

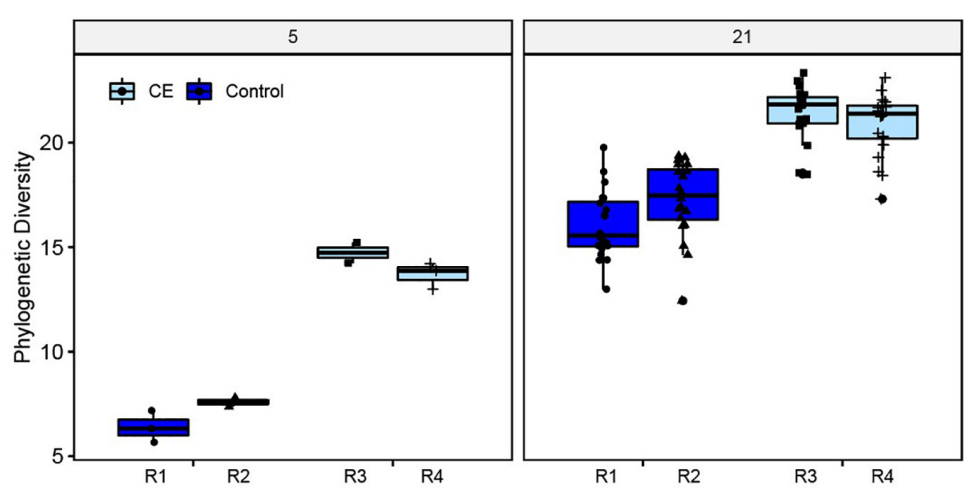

FIGURE 3 | Alpha (phylogenetic) diversity of cecal microbiota at day 5 ( $n=5$ broilers per intervention) and day 21 ( $n=40$ broilers per intervention), for the control (rooms (R) 1, 2) and intervention groups (rooms (R) 3, 4). 


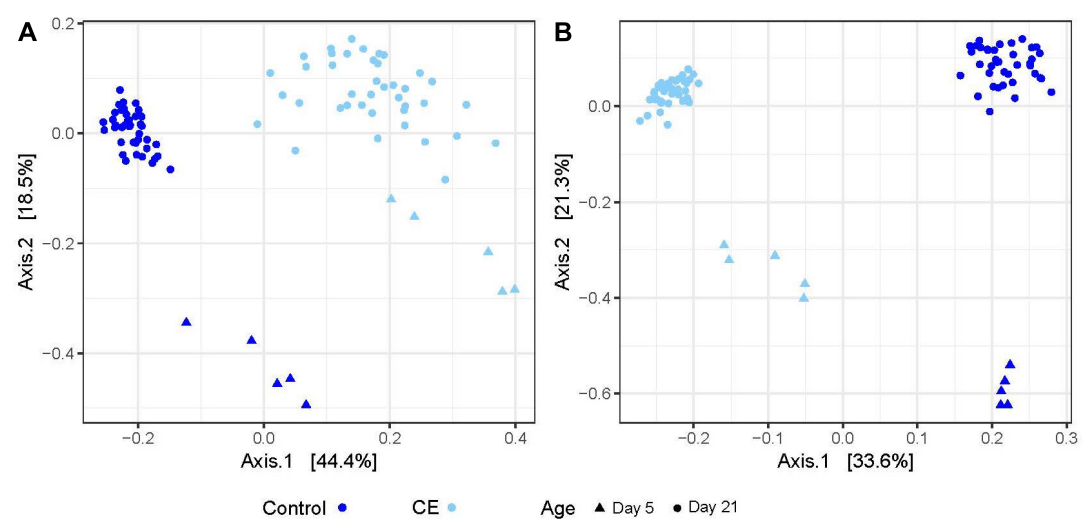

FIGURE 4 | Principal coordinate analysis (PCoA) of microbiota composition based on weighted UniFrac (A) and unweighted UniFrac (B) distances between control (dark blue) and CE (light blue) groups. Different symbols indicate different sampling days: triangles are samples of day 5 , and circles are samples of day 21.

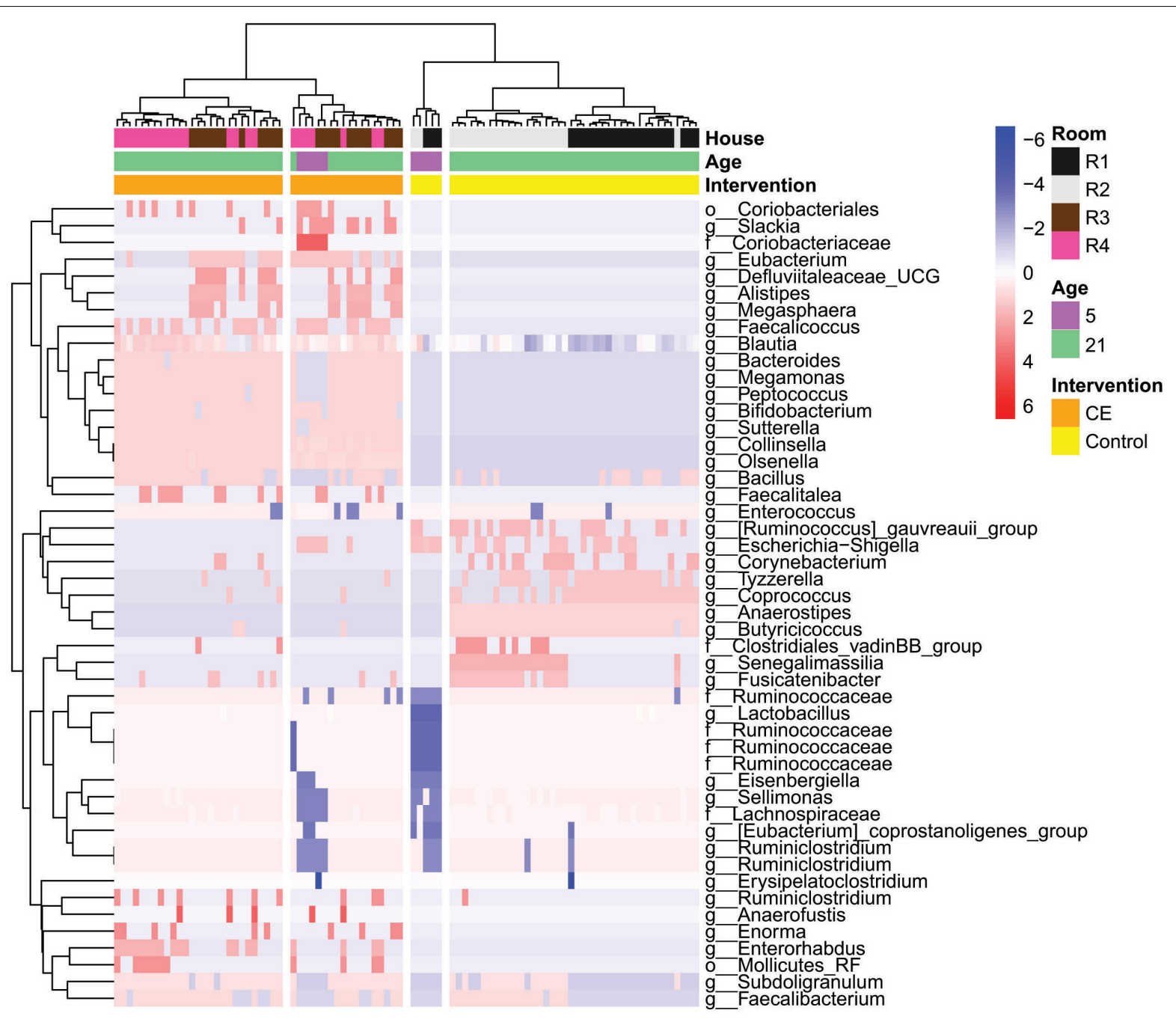

FIGURE 5 | Heatmap representing the abundance of amplicon sequence variants (ASVs) in all individual broiler chickens analyzed ( $n=90$ ). Only ASVs that are significantly different at day 5 and day 21 between CE and control are shown (Wilcoxon rank-sum test, adjusted $p$-values are corrected $p$-values for multiple testing, Benjamini-Hochberg, $p<0.05)$. Each red, white, or blue rectangle represents the relative abundance of a genus in an individual broiler. Clustering of broilers is based on Ward's minimum variance method and based on weighted UniFrac distances matrix. 
TABLE 2 | Relative abundance and standard deviation (SD) of genera that were present in the CE product, and the significantly different relative abundance in cecal content of CE broilers vs. control broilers at day $5(n=10)$ and $21(n=80)$.

\begin{tabular}{|c|c|c|c|c|c|c|c|c|}
\hline \multicolumn{3}{|c|}{ Relative abundance CE product } & \multicolumn{6}{|c|}{ Change in relative abundance CE vs. control broilers } \\
\hline \multirow[b]{2}{*}{ Genera } & \multirow[b]{2}{*}{ Relative abundance (\%) } & \multirow[b]{2}{*}{ SD (\%) } & \multicolumn{3}{|c|}{ Day 5 Relative abundance (\%) } & \multicolumn{3}{|c|}{ Day 21 Relative abundance (\%) } \\
\hline & & & Control & $C E$ & $p$-value & Control & $C E$ & $p$-value \\
\hline [Eubacterium] coprostanoligenes group & 0.65 & 0.22 & & & & 0.70 & 1.11 & $8.05 \times 10^{-07}$ \\
\hline Bacteroides & 0.47 & 0.06 & & & & - & 1.12 & $9.06 \times 10^{-15}$ \\
\hline Blautia & 0.30 & 0.09 & & & & 18.48 & 6.64 & $2.75 \times 10^{-12}$ \\
\hline Candidatus_Soleaferrea & 0.39 & 0.06 & & & & & & \\
\hline Clostridium sensu stricto 1 & 2.77 & 0.45 & 14.67 & 0.72 & 0.03 & 0.04 & - & 0.04 \\
\hline Clostridium sensu stricto 2 & 0.72 & 0.12 & & & & & & \\
\hline Collinsella & 0.53 & 0.07 & - & 12.98 & 0.03 & - & 4.28 & $3.34 \times 10^{-15}$ \\
\hline Enterococcus & 10.80 & 1.07 & 31.76 & 13.40 & 0.03 & 0.46 & 0.94 & $2.67 \times 10^{-03}$ \\
\hline Erysipelatoclostridium & 2.53 & 0.09 & & & 0.03 & 1.84 & 0.99 & 0.03 \\
\hline Escherichia-Shigella & 0.57 & 0.02 & 15.09 & 0.99 & 0.03 & 0.09 & $3.72 \times 10^{-03}$ & $2.04 \times 10^{-04}$ \\
\hline Eubacterium & 0.66 & 0.04 & - & 3.31 & 0.03 & - & 0.20 & $2.22 \times 10^{-07}$ \\
\hline Flavonifractor & 1.02 & 0.14 & - & 0.79 & 0.03 & & & \\
\hline Lachnoclostridium & 9.78 & 0.93 & - & 1.77 & 0.03 & & & \\
\hline Lactobacillus & 14.96 & 1.33 & - & 10.77 & 0.03 & & & \\
\hline Megamonas & 1.55 & 0.56 & & & & - & 10.36 & $3.34 \times 10^{-15}$ \\
\hline Megasphaera & 3.30 & 0.74 & & & & - & 0.27 & $3.82 \times 10^{-05}$ \\
\hline Negativicoccus & 3.62 & 0.66 & & & & & & \\
\hline Oscillibacter & 1.94 & 0.18 & & & & & & \\
\hline Peptostreptococcus & 30.97 & 4.04 & & & & & & \\
\hline Sellimonas & 1.31 & 0.38 & & & & 1.14 & 0.64 & $2.60 \times 10^{-04}$ \\
\hline Slackia & 0.34 & 0.09 & & & & - & 0.03 & $7.57 \times 10^{-03}$ \\
\hline Sutterella & 1.76 & 0.21 & & & & - & 0.99 & $3.34 \times 10^{-15}$ \\
\hline uncultured & 4.45 & 3.56 & & & & & & \\
\hline Unknown & 0.08 & 0.09 & & & & & & \\
\hline
\end{tabular}

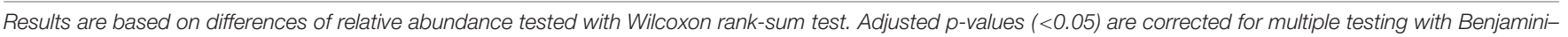
Hochberg $(\mathrm{BH})$. - = not detected.

TABLE 3 | Transmission coefficients ( $\beta_{\text {within }}$ and $\beta_{\text {between }}, 95 \% \mathrm{Cl}$ ) using an SI-model for transmission based on the proportion of excreting birds (model 1 ) and the accumulative excretion time (model 2).

Transmission coefficient $(\beta, 95 \% \mathrm{Cl})$

\begin{tabular}{|c|c|c|c|c|}
\hline & Unit $^{\star}$ & $\beta_{\text {within }}(95 \% \mathrm{Cl})$ & $\beta_{\text {between }}(95 \% \mathrm{Cl})$ & AIC \\
\hline Model 1 proportion excreting birds & day $^{-1}$ & $1.31(1.07-1.59)$ & $0.03(0.02-0.04)$ & 438.1 \\
\hline Model 2 accumulative excretion time & day $^{-2}$ & $6.12 \times 10^{-2}\left(4.78 \times 10^{-2}-7.64 \times 10^{-2}\right)$ & $3.28 \times 10^{-4}\left(2.41 \times 10^{-4}-4.32 \times 10^{-4}\right)$ & 402.1 \\
\hline
\end{tabular}

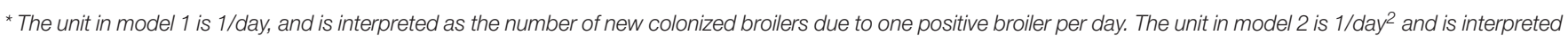
as the number of new colonized broilers caused by each day that one positive broiler has been excreting.

to -0.43$)$. Mean E. coli levels in cecal content did not differ between rooms or pens.

\section{DISCUSSION}

The supply of CE product via drinking water from day of hatch until day 7 prevented colonization of broilers with ESBL-producing E. coli after challenge of seeder birds. In the control group, $93.5 \%$ of the broilers were colonized at the end of the experiment. These results are in line with earlier experiments within isolators, in which a continuous supply of CE product during the first 14 days was able to prevent colonization (Dame-Korevaar et al., 2020). In the isolators in which at least one bird was colonized with ESBLproducing E. coli, application of $\mathrm{CE}$ products reduced the rate of colonization, decreased excretion (CFU/g) and reduced transmission, as previously shown in studies applying a single supply of CE (Hofacre et al., 2002; Nuotio et al., 2013; Ceccarelli et al., 2017; Methner et al., 2019). The enhanced effect of CE product found in this study compared to these earlier studies could have resulted from the prolonged supply, the longer period between start of $\mathrm{CE}$ product and exposure 
TABLE 4 | Parameter estimates for cecal content levels at day $21\left(\log _{10}\right.$ CFU/g cecal content, $95 \% \mathrm{Cl})$ of CTX-M-1-E. coli $(n=75)$ using a linear regression model.

\begin{tabular}{lc}
\hline Variable & Estimate CTX-M-1-E. coli $(\mathbf{9 5} \% \mathbf{C l})$ \\
\hline Room 1, pen Seeder/C1 (intercept) & $3.95(0.93$ to 6.98$)$ \\
Room 2 & $-0.52(-0.91$ to -0.13$)$ \\
Pen C2 & $-1.17(-1.91$ to -0.43$)$ \\
Pen C2 2 & $-2.28(-3.23$ to -1.33$)$ \\
Pen C2 & $-1.85(-2.95$ to -0.75$)$ \\
Pen C2 & $-2.01(-2.64$ to -1.39$)$ \\
Pen C2 & $-0.86(-1.40$ to -0.33$)$ \\
Pen C2 & $-0.59(-1.52$ to 0.33$)$ \\
Pen C2 & $0.34(-0.77$ to 1.46$)$ \\
Pen C2 & $-2.69(-3.49$ to -1.88$)$ \\
Bodyweight day of hatch (day 0) & $0.08(0.01$ to 0.15$)$ \\
\hline
\end{tabular}

to ESBL-producing E. coli, or the moment of challenge with ESBL-producing $E$. coli and the low challenge dose used in our study.

The microbiota composition was more diverse in the $\mathrm{CE}$ broilers than in the control broilers on day 5 and 21. This supports the hypothesis that microbial diversity plays a role in preventing colonization. Successful CE of ESBL-producing E. coli by specific genera being present in the CE broilers could also have prevented colonization. Intestinal colonization with microbiota of adult donor hens is associated with increased resistance against colonization, e.g., with Salmonella (Varmuzova et al., 2016). In a study where newly hatched layer chicks were exposed to an adult hen, transfer of microbiota occurred within $24 \mathrm{~h}$ of contact and a 1-3 days longer contact period resulted in an even more developed chick microbiota (Kubasova et al., 2019). In our study, supplying a CE product derived from intestinal bacteria of adult chickens possibly increased resistance and prevented colonization with ESBL-producing E. coli. The higher diversity observed in broilers at day 5 was maintained during the experiment. At day 21, 2 weeks after the last supply of the CE product, the intestinal microbiota composition was still more diverse in the CE broilers. Next to genera identified in the CE product, also other genera were found to be different between $\mathrm{CE}$ and control groups, indicating that the intestinal microbiota of CE broilers was early and persistently different compared to the composition of the microbiota as observed in the control broilers.

Direct competition between specific bacteria and inoculated E. coli in CE broilers might have played a role in preventing colonization, including competition for binding sites or limiting nutrients (Nurmi et al., 1992; Callaway et al., 2008). This could be related also with the production of antimicrobial compounds, including volatile fatty acids, inhibiting or eliminating species that compete for the same niche (Callaway et al., 2008). Some genera were present exclusively in the $\mathrm{CE}$ product and in CE broilers, but not in control broilers (Table 2). Due to competition, these genera might have prevented colonization. Next to preventing colonization with E. coli, CE products have shown to prevent or reduce colonization of different bacteria, e.g., Salmonella (Nakamura et al., 2002; Ferreira et al., 2003;
Luoma et al., 2017; Markazi et al., 2018) and Campylobacter (Schneitz and Hakkinen, 2016).

In the control groups compartmentalization resulted in a significantly lower transmission between pens than within pens. Transmission between pens shows that environmental transmission can occur and presence of ESBL/pAmpC-producing bacteria in litter, air or dust plays a role in transmission (Friese et al., 2013; Laube et al., 2013, 2014; Blaak et al., 2014, 2015; Daehre et al., 2018). Delayed transmission as result of compartmentalization has been described for other pathogens (van Bunnik et al., 2012; Dekker et al., 2013). The effect of compartmentalization can be two-fold: the physical barrier prevents direct contact between broilers, and next to that, during the time needed for transmission between pens to occur, the microbiota of the susceptible broilers might develop further, making it more difficult for ESBLproducing $E$. coli to colonize. In chickens, microbiota in the first week of life contains Enterobacteriaceae (Videnska et al., 2014; Ballou et al., 2016; Jurburg et al., 2019) suggesting that E. coli can easily colonize during the first week. Older bird might get less susceptible for colonization (Dame-Korevaar et al., 2020), however in our study colonization with ESBLproducing E. coli still occurred at 21 days of life, maybe as a result of accumulation of excreted ESBL-producing E. coli in the environment. Once transmission between pens occurred, transmission within pens followed rapidly. In room 1, within S/C1-pen transmission occurred very fast: all except one bird were positive at the first sampling after challenge. Therefore, this pen could not be included in the estimation of within pen transmission.

Estimation of the transmission coefficients was done using the proportion of excreting birds (model 1) and the accumulative excretion time (model 2 ). The model including excretion time fitted better to the observed data, indicating that accumulation of ESBL-producing E. coli in the environment most likely plays a role in the transmission within a flock. This increased infectivity by accumulation of the bacteria has been modeled also for other pathogens in pigs and chicken (Lurette et al., 2008; Dekker et al., 2013; van Bunnik et al., 2014). In our model, environmental accumulation is assumed to be unlimited, whereas in practice it is likely that there is a certain maximum, as postulated by van Bunnik et al. (2014) that assumed the force of infection to be limited by a maximum exposure capacity for recipient animals. However, in our study models including a maximum exposure capacity rendered a model that did not converge, which might indicate that the maximum exposure capacity was not yet reached at the end of the experiment.

In poultry practice, the interventions studied in this experiment could be used to control the spread of ESBLproducing E. coli. CE product could be supplied on the farm via the drinking water system. Supply should be done as soon as possible after hatching, before exposure to ESBLproducing E. coli occurs. In this study compartmentalization, including separation of feed- and water systems and strict hygiene measures, reduced transmission of ESBL-producing E. coli but could not prevent it. In practice, with less strict hygiene measures, the effects might be smaller. 


\section{CONCLUSION}

Overall, our study shows that CE is a useful intervention tool to prevent colonization of ESBL-producing bacteria after challenge with a low dose in the first week in a broiler flock. Transmission within a flock could be delayed by compartmentalization, however as soon as ESBL-producing bacteria are excreted and accumulate in the environment spread to other birds seems inevitable. Therefore, compartmentalization of large flocks into smaller groups of birds, which is for instance more common in breeding flocks at higher levels of the broiler production chain, could be combined to enhance the efficacy of other interventions. CE products could be supplied to young chicks after hatching at different levels of the broiler production pyramid to prevent colonization of birds. The insights provided by this study may provide a basis for further developments toward practically applicable measures to further reduce antimicrobial resistance in poultry.

\section{DATA AVAILABILITY STATEMENT}

Raw sequence data were deposited into the Sequence Read Archive (SRA) at the NCBI, under accession number PRJNA647260.

\section{ETHICS STATEMENT}

The animal study was reviewed and approved by Dutch Central Authority for Scientific Procedures on Animals and the

\section{REFERENCES}

Agerso, Y., Jensen, J. D., Hasman, H., and Pedersen, K. (2014). Spread of extended spectrum cephalosporinase-producing Escherichia coli clones and plasmids from parent animals to broilers and to broiler meat in a production without use of cephalosporins. Foodborne Pathog. Dis. 11, 740-746. doi: 10.1089/fpd. 2014.1742

Anderson, M. J. (2001). A new method for non-parametric multivariate analysis of variance. Austral. Ecol. 26, 32-46. doi: 10.1111/j.1442-9993.2001.01070.pp.x

Apostolakos, I., Mughini-Gras, L., Fasolato, L., and Piccirillo, A. (2019). Assessing the occurrence and transfer dynamics of ESBL/pAmpC-producing Escherichia coli across the broiler production pyramid. PLoS One 14:e0217174. doi: 10.1371/ journal.pone.0217174

Ballou, A. L., Ali, R. A., Mendoza, M. A., Ellis, J. C., Hassan, H. M., Croom, W. J., et al. (2016). Development of the chick microbiome: how early exposure influences future microbial diversity. Front. Vet. Sci. 3:2. doi: 10.3389/fvets. 2016.00002

Blaak, H., Hamidjaja, R. A., van Hoek, A. H., de Heer, L., de Roda Husman, R. H., and Schets, F. M. (2014). Detection of extended-spectrum beta-lactamase (ESBL)-producing Escherichia coli on flies at poultry farms. Appl. Environ. Microbiol. 80, 239-246. doi: 10.1128/AEM.02616-13

Blaak, H., van, H. A., Hamidjaja, R., van der, P. R., Kerkhof-de, H. L., de Roda, H. A., et al. (2015). Distribution, numbers, and diversity of ESBL-producing E. coli in the poultry farm environment. PLoS One 10:e0135402. doi: 10.1371/ journal.pone.0135402

Callaway, T. R., Edrington, T. S., Anderson, R. C., Harvey, R. B., Genovese, K. J., Kennedy, C. N., et al. (2008). Probiotics, prebiotics and competitive exclusion for prophylaxis against bacterial disease. Anim. Health Res. Rev. 9, 217-225. doi: $10.1017 /$ S1466252308001540
Animal Experiments Committee of Utrecht University (Utrecht, Netherlands) under registration number AVD108002015314.

\section{AUTHOR CONTRIBUTIONS}

AD, EF, JvdG, FV, DC, DM, and AS: experiment design. AD and FV: experiment execution. AD, JK, and EF: data analysis. AD and JK: manuscript writing. All authors discussed, read, contributed to, and approved the final manuscript.

\section{FUNDING}

This work was funded by the 1 Health 4 Food Public Private Partnership project: Reduction of ESBLs: evaluation of ESBL interventions (Grant number TKI-AF-14210). Microbiota analyses was funded through the ZonMW project MITAR (541001005).

\section{ACKNOWLEDGMENTS}

We thank Mirlin Spaninks (Utrecht University), Joop Testerink and Kees Veldman (Wageningen Bioveterinary Research), Ineke Heikamp-de Jong and Merlijn van Gaal (Wageningen University \& Research) for their technical assistance in the microbial analysis, the animal caretakers, Evelien Rondeel, Celine Jurjens, and Sam Stokvis (Utrecht University) for their assistance during the animal trials and the poultry facility for supplying the broilers.

Ceccarelli, D., van Essen-Zandbergen, A., Smid, B., Veldman, K. T., Boender, G. J., Fischer, E. A. J., et al. (2017). Competitive exclusion reduces transmission and excretion of extended-spectrum-beta-lactamase-producing Escherichia coli in broilers. Appl. Environ. Microbiol. 83:3439.

Daehre, K., Projahn, M., Semmler, T., Roesler, U., and Friese, A. (2018). Extendedspectrum beta-lactamase-/AmpC beta-lactamase-producing Enterobacteriaceae in broiler farms: transmission dynamics at farm level. Microb. Drug Resist. 24, 511-518. doi: 10.1089/mdr.2017.0150

Dame-Korevaar, A., Fischer, E. A. J., van der Goot, J., Stegeman, A., and Mevius, D. (2019a). Transmission routes of ESBL/pAmpC producing bacteria in the broiler production pyramid, a literature review. Prev. Vet. Med. 162, 136-150. doi: 10.1016/j.prevetmed.2018.12.002

Dame-Korevaar, A., Fischer, E. A. J., van der Goot, J., Velkers, F., van den Broek, J., Veldman, K., et al. (2019b). Effect of challenge dose of plasmid-mediated extended-spectrum $\beta$-lactamase and AmpC $\beta$-lactamase producing Escherichia coli on time-until-colonization and level of excretion in young broilers. Vet. Microbiol. 239:108446. doi: 10.1016/j.vetmic.2019.108446

Dame-Korevaar, A., Fischer, E. A. J., van der Goot, J., Velkers, F., Ceccarelli, D., Mevius, D., et al. (2020). Early life supply of competitive exclusion products reduces colonization of extended spectrum beta-lactamase producing Escherichia coli in broilers. Poult. Sci. 99, 4052-4064. doi: 10.1016/j.psj.2020.04. 025

Dekker, N., Bouma, A., Daemen, I., Klinkenberg, D., van Leengoed, L., Wagenaar, J. A., et al. (2013). Effect of spatial separation of pigs on spread of Streptococcus suis serotype 9. PLoS One 8:e61339. doi: 10.1371/journal.pone.0061339

Dierikx, C., van Essen-Zandbergen, A., Veldman, K., Smith, H., and Mevius, D. (2010). Increased detection of extended spectrum beta-lactamase producing Salmonella enterica and Escherichia coli isolates from poultry. Vet. Microbiol. 145, 273-278. doi: 10.1016/j.vetmic.2010.03.019 
Dierikx, C. M., Van der Goot, J. A., Smith, H. E., Kant, A., and Mevius, D. J. (2013). Presence of ESBL/AmpC -producing Escherichia coli in the broiler production pyramid: a descriptive study. PLoS One 8:e79005. doi: 10.1371/journal.pone. 0079005

Donaldson, E. E., Stanley, D., Hughes, R. J., and Moore, R. J. (2017). The timecourse of broiler intestinal microbiota development after administration of cecal contents to incubating eggs. PeerJ. 5:e3587. doi: 10.7717/peerj.3587

Faith, D. P. (2007). The role of the phylogenetic diversity measure, PD, in bio-informatics: getting the definition right. Evol. Bioinform. Online 2, 277-283.

Ferreira, A. J., Ferreira, C. S., Knobl, T., Moreno, A. M., Bacarro, M. R., Chen, M., et al. (2003). Comparison of three commercial competitive-exclusion products for controlling Salmonella colonization of broilers in Brazil. J. Food Prot. 66, 490-492. doi: 10.4315/0362-028x-66.3.490

Friese, A., Schulz, J., Laube, H., von Salviati, C., Hartung, J., and Roesler, U. (2013). Faecal occurrence and emissions of livestock-associated methicillin-resistant Staphylococcus aureus (laMRSA) and ESbl/AmpC-producing E. coli from animal farms in Germany. Berl Munch Tierarztl Wochenschr 126, 175-180.

Hesp, A., Veldman, K., van der Goot, J., Mevius, D., and van Schaik, G. (2019). Monitoring antimicrobial resistance trends in commensal Escherichia coli from livestock, the Netherlands, 1998 to 2016. Euro. Surveill 24:1800438. doi: 10. 2807/1560-7917.ES.2019.24.25.1800438

Hofacre, C. L., Johnson, A. C., Kelly, B. J., and Froyman, R. (2002). Effect of a commercial competitive exclusion culture on reduction of colonization of an antibiotic-resistant pathogenic Escherichia coli in day-old broiler chickens. Avian. Dis. 46, 198-202. doi: 10.1637/0005-2086(2002)046[0198:eoacce]2. $0 . \mathrm{co} ; 2$

Jett, B. D., Hatter, K. L., Huycke, M. M., and Gilmore, M. S. (1997). Simplified agar plate method for quantifying viable bacteria. BioTechniques 23, 648-650. doi: $10.2144 / 97234 \mathrm{bm} 22$

Jones, E. M., Snow, L. C., Carrique-Mas, J. J., Gosling, R. J., Clouting, C., and Davies, R. H. (2013). Risk factors for antimicrobial resistance in Escherichia coli found in GB turkey flocks. Vet. Rec. 173:422. doi: 10.1136/vr.101759

Jurburg, S. D., Brouwer, M. S. M., Ceccarelli, D., van der Goot, J., Jansman, A. J. M., and Bossers, A. (2019). Patterns of community assembly in the developing chicken microbiome reveal rapid primary succession. Microbiologyopen 8:e00821. doi: 10.1002/mbo3.821

Kalbfleisch, J. D., and Prentice, R. L. (2002). The Statistical Analysis of Failure Time Data, 2nd Edn. New York, NY: John Wiley and Sons.

Kers, J. G., Velkers, F. C., Fischer, E. A. J., Hermes, G. D. A., Lamot, D. M., Stegeman, J. A., et al. (2019). Take care of the environment: housing conditions affect the interplay of nutritional interventions and intestinal microbiota in broiler chickens. Anim. Microbiome 1:10. doi: 10.1186/s42523-019-0009-z

Klinkenberg, D., de Bree, J., Laevens, H., and de Jong, M. C. M. (2002). Withinand between-pen transmission of classical swine fever virus: a new method to estimate the basic reproduction ratio from transmission experiments. Epidemiol. Infect 128, 293-299. doi: 10.1017/s0950268801006537

Kubasova, T., Kollarcikova, M., Crhanova, M., Karasova, D., Cejkova, D., Sebkova, A., et al. (2019). Contact with adult hen affects development of caecal microbiota in newly hatched chicks. PLoS One 14:e0212446. doi: 10.1371/ journal.pone. 0212446

Laube, H., Friese, A., von Salviati, C., Guerra, B., Kasbohrer, A., Kreienbrock, L., et al. (2013). Longitudinal monitoring of extended-spectrum-betalactamase/AmpC-producing Escherichia coli at German broiler chicken fattening farms. Appl. Environ. Microbiol 79, 4815-4820. doi: 10.1128/AEM. 00856- 13

Laube, H., Friese, A., von Salviati, C., Guerra, B., and Rosler, U. (2014). Transmission of ESBL/AmpC-producing Escherichia coli from broiler chicken farms to surrounding areas. Vet. Microbiol. 172, 519-527. doi: 10.1016/j.vetmic. 2014.06.008

Lozupone, C. A., Hamady, M., Kelley, S. T., and Knight, R. (2007). Quantitative and qualitative beta diversity measures lead to different insights into factors that structure microbial communities. Appl. Environ. Microbiol. 73, 1576-1585. doi: 10.1128/aem.01996-06

Luoma, A., Markazi, A., Shanmugasundaram, R., Murugesan, G. R., Mohnl, M., and Selvaraj, R. (2017). Effect of synbiotic supplementation on layer production and cecal Salmonella load during a Salmonella challenge. Poult. Sci. 96, 42084216. doi: $10.3382 / \mathrm{ps} / \mathrm{pex} 251$
Lurette, A., Belloc, C., Touzeau, S., Hoch, T., Ezanno, P., Seegers, H., et al. (2008). Modelling Salmonella spread within a farrow-to-finish pig herd. Vet. Res. 39:49. doi: 10.1051/vetres:2008026

Markazi, A., Luoma, A., Shanmugasundaram, R., Mohnl, M., Raj Murugesan, G., and Selvaraj, R. (2018). Effects of drinking water synbiotic supplementation in laying hens challenged with Salmonella. Poult. Sci. 97, 3510-3518. doi: 10.3382/ ps/pey234

Methner, U., Friese, A., and Rosler, U. (2019). Competitive exclusion: a tool to combat extended-spectrum beta-lactamase-producing Escherichia coli strains in chickens. Res. Vet. Sci. 123, 124-128. doi: 10.1016/j.rvsc.2019.01.003

Nakamura, A., Ota, Y., Mizukami, A., Ito, T., Ngwai, Y. B., and Adachi, Y. (2002). Evaluation of aviguard, a commercial competitive exclusion product for efficacy and after-effect on the antibody response of chicks to Salmonella. Poult. Sci. 81, 1653-1660. doi: 10.1093/ps/81.11.1653

Nilsson, O., Borjesson, S., Landen, A., and Bengtsson, B. (2014). Vertical transmission of Escherichia coli carrying plasmid-mediated AmpC (pAmpC) through the broiler production pyramid. J. Antimicrob. Chemother. 69, 14971500. doi: 10.1093/jac/dku030

Nuotio, L., Schneitz, C., and Nilsson, O. (2013). Effect of competitive exclusion in reducing the occurrence of Escherichia coli producing extended-spectrum betalactamases in the ceca of broiler chicks. Poult. Sci. 92, 250-254. doi: 10.3382/ps. 2012-02575

Nurmi, E., Nuotio, L., and Schneitz, C. (1992). The competitive exclusion concept: development and future. Int. J. Food Microbiol. 15, 237-240. doi: 10.1016/01681605(92)90054-7

Projahn, M., Daehre, K., Semmler, T., Guenther, S., Roesler, U., and Friese, A. (2018). Environmental adaptation and vertical dissemination of ESBL/pAmpC-producing Escherichia coli in an integrated broiler production chain in the absence of an antibiotic treatment. Microb. Biotechnol. 11, 1017-1026. doi: 10.1111/1751-7915.13040

Quast, C., Pruesse, E., Yilmaz, P., Gerken, J., Schweer, T., Yarza, P., et al. (2013). The SILVA ribosomal RNA gene database project: improved data processing and web-based tools. Nucleic Acids Res. 41:590. doi: 10.1093/nar/gks1219

Ramiro-Garcia, J., Hermes, G. D. A., Giatsis, C., Sipkema, D., Zoetendal, E. G., Schaap, P. J., et al. (2016). NG-Tax, a highly accurate and validated pipeline for analysis of 16S rRNA amplicons from complex biomes. F1000Res 5:1791. doi: 10.12688/f1000research.9227.2

Roth, N., Mayrhofer, S., Gierus, M., Weingut, C., Schwarz, C., Doupovec, B., et al. (2017). Effect of an organic acids based feed additive and enrofloxacin on the prevalence of antibiotic-resistant E. coli in cecum of broilers. Poult. Sci. 96, 4053-4060. doi: $10.3382 / \mathrm{ps} / \mathrm{pex} 232$

RStudio Team (2016). RStudio: Integrated Development for R. Boston, MA: RStudio.

Saliu, E. M., Vahjen, W., and Zentek, J. (2017). Types and prevalence of extended-spectrum beta-lactamase producing Enterobacteriaceae in poultry. Anim. Health Res. Rev. 18, 46-57. doi: 10.1017/S146625231700 0020

Schneitz, C., and Hakkinen, M. (2016). The efficacy of a commercial competitive exclusion product on Campylobacter colonization in broiler chickens in a 5-week pilot-scale study. Poult. Sci. 95, 1125-1128. doi: 10.3382/ps/ pew020

Schokker, D., Jansman, A. J., Veninga, G., de Bruin, N., Vastenhouw, S. A., de Bree, F. M., et al. (2017). Perturbation of microbiota in one-day old broiler chickens with antibiotic for 24 hours negatively affects intestinal immune development. BMC Genomics 18:241. doi: 10.1186/s12864-017-3625-6

van Bunnik, B. A., Katsma, W. E., Wagenaar, J. A., Jacobs-Reitsma, W. F., and de Jong, M. C. (2012). Acidification of drinking water inhibits indirect transmission, but not direct transmission of campylobacter between broilers. Prev. Vet. Med. 105, 315-319. doi: 10.1016/j.prevetmed.2012. 03.007

van Bunnik, B. A., Ssematimba, A., Hagenaars, T. J., Nodelijk, G., Haverkate, M. R., Bonten, M. J., et al. (2014). Small distances can keep bacteria at bay for days. Proc. Natl. Acad. Sci. U.S.A. 111, 3556-3560. doi: 10.1073/pnas.13100 43111

Varmuzova, K., Kubasova, T., Davidova-Gerzova, L., Sisak, F., Havlickova, H., Sebkova, A., et al. (2016). Composition of gut microbiota influences resistance of newly hatched chickens to Salmonella enteritidis infection. Front. Microbiol. 7:957. doi: $10.3389 /$ fmicb.2016.00957 
Velthuis, A. G., Bouma, A., Katsma, W. E., Nodelijk, G., and De Jong, M. C. (2007). Design and analysis of small-scale transmission experiments with animals. Epidemiol. Infect 135, 202-217. doi: 10.1017/s0950268806 00673x

Videnska, P., Sedlar, K., Lukac, M., Faldynova, M., Gerzova, L., Cejkova, D., et al. (2014). Succession and replacement of bacterial populations in the caecum of egg laying hens over their whole life. PLoS One 9:e115142. doi: 10.1371/journal. pone. 0115142

Zurfluh, K., Jakobi, G., Stephan, R., Hachler, H., and Nuesch-Inderbinen, M. (2014a). Replicon typing of plasmids carrying bla CTX-M-1 in Enterobacteriaceae of animal, environmental and human origin. Front. Microbiol. 5:555. doi: 10.3389/fmicb.2014.00555

Zurfluh, K., Wang, J., Klumpp, J., Nuesch-Inderbinen, M., Fanning, S., and Stephan, R. (2014b). Vertical transmission of highly similar bla CTX-M-1harboring IncI1 plasmids in Escherichia coli with different MLST types in the poultry production pyramid. Front. Microbiol. 5:519. doi: 10.3389/fmicb.2014. 00519
Disclaimer:The views expressed are purely those of the author(s) and may not in any circumstances be regarded as stating an official position of the European Commission.

Conflict of Interest: DC is currently employed by the Research Executive Agency.

The remaining authors declare that the research was conducted in the absence of any commercial or financial relationships that could be construed as a potential conflict of interest.

Copyright (C) 2020 Dame-Korevaar, Kers, van der Goot, Velkers, Ceccarelli, Mevius, Stegeman and Fischer. This is an open-access article distributed under the terms of the Creative Commons Attribution License (CC BY). The use, distribution or reproduction in other forums is permitted, provided the original author(s) and the copyright owner(s) are credited and that the original publication in this journal is cited, in accordance with accepted academic practice. No use, distribution or reproduction is permitted which does not comply with these terms. 